EPJ Web of Conferences 58, 02003 (2013)

DOI: $10.1051 /$ epjconf/20135802003

(C) Owned by the authors, published by EDP Sciences, 2013

\title{
Time Solutions and Symmetries in Extended Gravity Quantum Cosmology
}

\author{
Salvatore Capozziello $0^{1,2}$ \\ ${ }^{1}$ Dipartimento di Scienze Fisiche, Università di Napoli Federico II \\ ${ }^{2}$ INFN sez. di Napoli Compl. Univ. di Monte S. Angelo, Edificio G, Via Cinthia, I-80126 - Napoli, Italy
}

\begin{abstract}
Minisuperspace models are taken into account searching for Noether symmetries. The existence of conserved quantities gives selection rule to recover classical behaviors in cosmic evolution according to the so called Hartle criterion, that allows to select correlated regions in the configuration space of dynamical variables. We show that such a statement works for general classes of gravity theories. Examples for nonminimally coupled and higher-order models are discussed.
\end{abstract}

\section{Introduction}

Different points of view can be assumed in order to deal with Quantum Cosmology (QC). It can be considered as the first step towards the construction of a complete theory of Quantum Gravity. Moreover, its goal is to find out the law of initial conditions from which our classical universe started its evolution. However, not only the conceptual difficulties, but also mathematical ones make QC difficult to handle. For example, the superspace of geometrodynamics [1] has infinite degrees of freedom so that it is not possible to fully integrate the Wheeler-De Witt (WDW) equation. Moreover, the Hilbert space of states describing universes is not available and then it is not clear how to interpret the solutions of WDW equation in the framework of probability theory [2] . Despite of these shortcomings, several results have been obtained and QC has become a sort of paradigm in theoretical physics. For example the infinite-dimensional superspace can be restricted to suitable finite-dimensional configuration spaces, the so- called minisuperspaces. In this case, the above mathematical difficulties can be circumvented since the WDW equation reduces to a partial differential equation and, in principle, can be integrated. The initial value problem can be approached in some simplified ways that give reasonable laws for initial conditions from which our classical universe could be started. However, it is better to stress that QC is not fully satisfactory in view of solving Quantum Gravity issues but is a useful working scheme despite of different interpretations of results. For example, the Hartle criterion [3] is an interpretative scheme for the solutions of the WDW equation. Hartle proposed to look for peaks of the wave function of the universe: if it is strongly peaked, we have correlations among the geometrical and matter degrees of freedom; if it is not peaked, correlations are lost. In the first case, the emergence of classical trajectories (i.e. universes) is expected. More precisely, the wave function of the universe can be written as

$$
\Psi\left[h_{i j}(x), \phi(x)\right] \sim e^{i m_{P}^{2} \mathcal{S}},
$$

This is an Open Access article distributed under the terms of the Creative Commons Attribution License 2.0, which permits unrestricted use, distribution, and reproduction in any medium, provided the original work is properly cited. 
where $h^{i j}$ are the components of the 3-metric of geometrodynamics, $\phi$ is a matter scalar field, $m_{P}$ is the Planck mass and $\mathcal{S} \equiv \mathcal{S}_{0}+m_{P}^{-2} \mathcal{S}_{1}+O\left(m_{P}^{-4}\right)$ is the action which can be expanded. We have to note that there is no normalization factor due to the lack of a probability interpretative scheme. Inserting $\mathcal{S}$ into the WDW equation (that we will derive below) and equating similar powers of $m_{P}$, one obtains the Hamilton-Jacobi equation for $\mathcal{S}_{0}$. Similarly, one gets equations for $\mathcal{S}_{1}, \mathcal{S}_{2}, \ldots$, which can be solved considering results of previous orders. We need only $\mathcal{S}_{0}$ to recover the semi-classical limit of QC [4]. If $S_{0}$ is a real number, we get oscillating WKB modes and the Hartle criterion is recovered since $\Psi$ is peaked on a phase-space region defined by

$$
\pi_{i j}=m_{P}^{2} \frac{\delta S_{0}}{\delta h^{i j}}, \quad \pi_{\phi}=m_{P}^{2} \frac{\delta S_{0}}{\delta \phi},
$$

where $\pi_{i j}$ and $\pi_{\phi}$ are classical momenta conjugates to $h^{i j}$ and $\phi$. The semi-classical region of superspace, where $\Psi$ has an oscillating structure, is the Lorentz one otherwise it is Euclidean. In the latter case, we have $S=i I$ and $\Psi \sim e^{-m_{P}^{2} I}$, where $I$ is the action for the Euclidean solutions of classical field equations (istantons). This scheme, at least at semiclassical level, solves the problem of initial conditions. Given an action $S_{0}$, Eqs.(2) imply $n$ free parameters (one for each dimension of the configuration space $\left.Q \equiv\left\{h^{i j}, \phi\right\}\right)$ and then $n$ first integrals of motion. The general solution of the field equations implies $2 n-1$ parameters (one for any Hamilton equation plus the energy constraint). As a consequence, the wave function is peaked on a subset of the general solution and the boundary conditions on the wave function imply initial conditions for the classical solutions. The issue is now to search for a method capable of selecting these constants of motion. We want to show that the existence of Noether symmetries implies a subset of the general solution of the WDW equation where the oscillating behaviors are selected. Viceversa, the Hartle criterion can be always related to a Noether symmetry and then to the classical trajectories. For classical trajectories, we mean solutions of the standard cosmological equations. In particular, we restrict the discussion to minisuperspace models but it is clear that it could work for the complete field theory as soon as the method could be extended to the whole superspace. Here we consider minisuperspace models coming from Extended Theories of Gravity (ETGs) that have recently become a sort of paradigm in the study of gravitational interaction [5]. The scheme consists, essentially, in adding higher-order curvature invariants and/or non-minimally coupled scalar fields into dynamics resulting from the effective action of Quantum Gravity. These contributions come from one-loop or higher-loop corrections in the high-curvature regimes [6]. The approach gained interest in cosmology due to the fact that ETGs naturally exhibit inflationary behaviors and could explain the dark energy behavior observed at present epoch [7, 8]. In this paper, we discuss the Noether Symmetry Approach [9] in order to select minisuperspace classical solutions. Examples in non-minimally coupled and higher-order cosmologies are given.

\section{The Noether Symmetry Approach}

As we said before, minisuperspaces are restrictions of geometrodynamics superspace. They are finitedimensional configuration spaces on which point-like Lagrangians can be defined. Cosmological models of physical interest can be defined on such minisuperspaces (e.g. Bianchi models). The Noether Symmetry Approach, as we will show, is extremely useful to select classical trajectories on minisuperspace models. The discussion can be specified by considering a Lagrangian $\mathcal{L}$ which is a function defined on the tangent space of configurations $T Q \equiv\left\{q_{i}, \dot{q}_{i}\right\}$. In this case, the vector field $X$ is

$$
X=\alpha^{i}(q) \frac{\partial}{\partial q^{i}}+\dot{\alpha}^{i}(q) \frac{\partial}{\partial \dot{q}^{i}},
$$


where dot means derivative with respect to $t$, and

$$
L_{X} \mathcal{L}=X \mathcal{L}=\alpha^{i}(q) \frac{\partial \mathcal{L}}{\partial q^{i}}+\dot{\alpha}^{i}(q) \frac{\partial \mathcal{L}}{\partial \dot{q}^{i}} .
$$

The condition

$$
L_{X} \mathcal{L}=0
$$

implies that the phase flux is conserved along $X$ : this means that a constant of motion exists for $\mathcal{L}$ and the Noether theorem holds. If (5) holds, from the Euler-Lagrange equations one gets

$$
\Sigma_{0}=\alpha^{i} \frac{\partial \mathcal{L}}{\partial \dot{q}^{i}}
$$

that is a constant of motion. Alternatively, using the Cartan one-form $\theta_{\mathcal{L}} \equiv \frac{\partial \mathcal{L}}{\partial \dot{q}^{i}} d q^{i}$ and defining the inner derivative $i_{X} \theta_{\mathcal{L}}=<\theta_{\mathcal{L}}, X>$, we get as above $i_{X} \theta_{\mathcal{L}}=\Sigma_{0}$, when condition (5) holds. This representation is useful to identify cyclic variables. Using a point transformation on vector field (3), it is possible to get

$$
\tilde{X}=\left(i_{X} d Q^{k}\right) \frac{\partial}{\partial Q^{k}}+\left[\frac{d}{d t}\left(i_{X} d Q^{k}\right)\right] \frac{\partial}{\partial \dot{Q}^{k}} .
$$

If $X$ is a symmetry also $\tilde{X}$ has this property, then it is always possible to choose a coordinate transformation so that

$$
i_{X} d Q^{1}=1, \quad i_{X} d Q^{i}=0, \quad i \neq 1,
$$

and then

$$
\tilde{X}=\frac{\partial}{\partial Q^{1}}, \quad \frac{\partial \tilde{\mathcal{L}}}{\partial Q^{1}}=0 .
$$

It is evident that $Q^{1}$ is the cyclic coordinate and the dynamics can be reduced. However, the change of coordinates is not unique and a clever choice is always important. Furthermore, it is possible that more symmetries are found. In this case more cyclic variables exists. For example, if $X_{1}, X_{2}$ are the Noether vector fields and they commute, $\left[X_{1}, X_{2}\right]=0$, we obtain two cyclic coordinates. If they do not commute, $X_{3}=\left[X_{1}, X_{2}\right]$ is again a symmetry. If $X_{3}$ is independent of $X_{1}, X_{2}$ we can go on until the vector fields close the Lie algebra [10]. A reduction procedure by cyclic coordinates can be implemented in three steps: i) we choose a symmetry and obtain new coordinates as above. After this first reduction, we get a new Lagrangian $\tilde{\mathcal{L}}$ with a cyclic coordinate; ii) we search for new symmetries in this new space and apply the reduction technique until it is possible; iii) the process stops if we select a pure kinetic Lagrangian where all coordinates are cyclic. This case is not very common and often it is not physically relevant. Going back to the QC viewpoint, any symmetry selects a constant conjugate momentum since, by the Euler-Lagrange equations, we have

$$
\frac{\partial \tilde{\mathcal{L}}}{\partial Q^{i}}=0 \Longleftrightarrow \frac{\partial \tilde{\mathcal{L}}}{\partial \dot{Q}^{i}}=\Sigma_{i}
$$

Viceversa, the existence of a constant conjugate momentum means that a cyclic variable has to exist. In other words, a Noether symmetry exists. As in analytical mechanics, $\mathcal{L}$ can be set in the form

$$
\mathcal{L}=T\left(q^{i}, \dot{q}^{i}\right)-V\left(q^{i}\right)
$$


where $T$ is a positive-defined quadratic form in the $\dot{q}^{j}$ and $V\left(q^{i}\right)$ is a potential term. By the Legendre transformations

$$
\mathcal{H}=\pi_{j} \dot{q}^{j}-\mathcal{L}\left(q^{j}, \dot{q}^{j}\right), \quad \pi_{j}=\frac{\partial \mathcal{L}}{\partial \dot{q}^{j}},
$$

we get the Hamiltonian function and the conjugate momenta. Considering again the symmetry, the condition (5) and the vector field $X$ in Eq.(3) give a homogeneous polynomial of second degree in the velocities plus an inhomogeneous term in the $q^{j}$. Due to (5), such a polynomial has to be identically zero and then each coefficient must be independently zero. If $n$ is the dimension of the configuration space (i.e. the dimension of the minisuperspace), we get $\{1+n(n+1) / 2\}$ partial differential equations whose solutions assign the symmetry. Such a symmetry is over-determined and, if a solution exists, it is expressed in terms of integration constants. In the Hamiltonian formalism, we have

$$
\left[\Sigma_{j}, \mathcal{H}\right]=0, \quad 1 \leq j \leq m,
$$

as it must be for conserved momenta in quantum mechanics. The Hamiltonian has to satisfy the relation

$$
L_{\Gamma} \mathcal{H}=0 \text {, }
$$

to obtain a Noether symmetry. The vector $\Gamma$ is defined as

$$
\Gamma=\dot{q}^{i} \frac{\partial}{\partial q^{i}}+\ddot{q}^{i} \frac{\partial}{\partial \dot{q}^{i}} .
$$

These considerations can be applied to the minisuperspace models and to the interpretation of the wave function of the universe. By a straightforward canonical quantization procedure, we have

$$
\pi_{j} \longrightarrow \hat{\pi}_{j}=-i \partial_{j}, \quad \mathcal{H} \longrightarrow \hat{\mathcal{H}}\left(q^{j},-i \partial_{q^{j}}\right),
$$

The Hamiltonian constraint gives the WDW equation, so that if $\mid \Psi>$ is a state of the system (i.e. the wave function of the universe), dynamics is given by the WDW equation

$$
\mathcal{H} \mid \Psi>=0 .
$$

If a Noether symmetry exists, the reduction procedure outlined above can be applied and then, from (10) and (12), we get

$$
\pi_{1} \equiv \frac{\partial \mathcal{L}}{\partial \dot{Q}^{1}}=i_{X_{1}} \theta_{\mathcal{L}}=\Sigma_{1}, \quad \pi_{2} \equiv \frac{\partial \mathcal{L}}{\partial \dot{Q}^{2}}=i_{X_{2}} \theta_{\mathcal{L}}=\Sigma_{2}, \quad \ldots,
$$

depending on the number of Noether symmetries. After quantization, we have

$$
-i \partial_{1}\left|\Psi>=\Sigma_{1}\right| \Psi>, \quad-i \partial_{2}\left|\Psi>=\Sigma_{2}\right| \Psi>, \quad \ldots,
$$

which are nothing else but translations along the $Q^{j}$ axes singled out by the corresponding symmetries. Eqs. (19) can be immediately integrated and, being $\Sigma_{j}$ real constants, we obtain oscillatory behaviors for $\mid \Psi>$ in the directions of symmetries, i.e.

$$
\left|\Psi>=\sum_{j=1}^{m} e^{i \Sigma_{j} Q^{j}}\right| \chi\left(Q^{l}\right)>, \quad m<l \leq n,
$$

where $m$ is the number of symmetries, $l$ are the directions where symmetries do not exist, $n$ is the total dimension of minisuperspace. Viceversa, dynamics given by (17) can be reduced by (19) if and only if 
it is possible to define constant conjugate momenta as in (18), that is oscillatory behaviors of a subset of solutions $\mid \Psi>$ exist only if Noether symmetries exist for dynamics. The $m$ symmetries give first integrals of motion and then the possibility to select classical trajectories. In one and two-dimensional minisuperspaces, the existence of a Noether symmetry allows the complete solution of the problem and to get the full semi-classical limit of QC $[11,12]$. In conclusion, we can state that in the semiclassical limit of QC, the reduction procedure of dynamics, connected to the existence of Noether symmetries, allows to select subsets of the WDW equation solutions where oscillatory behaviors are found. This fact, in the framework of the Hartle interpretative criterion, gives conserved momenta and trajectories which can be interpreted as classical cosmological solutions. Vice-versa, if a subset of the WDW equation solution has an oscillatory behavior, conserved momenta exist due to the Noether symmetries. In other words, Noether symmetries select classical universes. In what follows, we will show that such a statement holds for general classes of minisuperspaces. In this sense, the Noether symmetries are a selection criterion for classical universes.

\section{Extended Minisuperspace Models}

In what follows, we shall give realizations of the above approach for minisuperspace cosmological models derived from ETGs. In particular, we give minisuperspace examples and obtain exact cosmological solutions for non-minimally coupled and higher-order theories.

\subsection{Scalar-Tensor Gravity Cosmologies}

Let us take into account a nonminimally coupled theory of gravity of the form

$$
\mathcal{S}=\int d^{4} x \sqrt{-g}\left[F(\phi) R+\frac{1}{2} g^{\mu v} \phi_{\mu} \phi_{v}-V(\phi)\right],
$$

where $F(\phi)$ and $V(\phi)$ are respectively the coupling and the potential of a scalar field [14]. We are using physical units $8 \pi G=c=\hbar=1$, so that the standard Einstein coupling is recovered for $F(\phi)=-1 / 2$. Let us restrict to a Friedmann-Robertson-Walker (FRW) minisuperspace. In this case, the Lagrangian in (21) becomes point-like, that is

$$
\mathcal{L}=6 a \dot{a}^{2} F+6 a^{2} \dot{a} \dot{F}-6 k a F+a^{3}\left[\frac{\dot{\phi}}{2}-V\right],
$$

in terms of the scale factor $a$. The configuration space of such a Lagrangian is $Q \equiv\{a, \phi\}$, i.e. a bidimensional minisuperspace. A Noether symmetry exists if (5) holds. In this case, it has to be

$$
X=\alpha \frac{\partial}{\partial a}+\beta \frac{\partial}{\partial \phi}+\dot{\alpha} \frac{\partial}{\partial \dot{a}}+\dot{\beta} \frac{\partial}{\partial \dot{\phi}},
$$

where $\alpha, \beta$ depend on $a, \phi$. This vector field acts on the $Q$ minisuperspace. The system of partial differential equation given by (5) is

$$
\begin{aligned}
& F(\phi)\left[\alpha+2 a \frac{\partial \alpha}{\partial a}\right]+a F^{\prime}(\phi)\left[\beta+a \frac{\partial \beta}{\partial a}\right]=0, \\
& 3 \alpha+12 F^{\prime}(\phi) \frac{\partial \alpha}{\partial \phi}+2 a \frac{\partial \beta}{\partial \phi}=0 \\
& a \beta F^{\prime \prime}(\phi)+\left[2 \alpha+a \frac{\partial \alpha}{\partial a}+\frac{\partial \beta}{\partial \phi}\right] F^{\prime}(\phi)+2 \frac{\partial \alpha}{\partial \phi} F(\phi)+\frac{a^{2}}{6} \frac{\partial \beta}{\partial a}=0, \\
& \left.3 \alpha V(\phi)+a \beta V^{\prime}(\phi)\right] a^{2}+6 k\left[\alpha F(\phi)+a \beta F^{\prime}(\phi)=0 .\right.
\end{aligned}
$$


Prime indicates the derivative with respect to $\phi$. The number of equations is 4 as it has to be, being $n=2$ the $Q$-dimension. Several solutions exist for this system [14]. They determine also the form of the model since the system (24)-(27) gives $\alpha, \beta, F(\phi)$ and $V(\phi)$. For example, if the spatial curvature is $k=0$, a solution is

$$
\alpha=-\frac{2}{3} p(s) \beta_{0} a^{s+1} \phi^{m(s)-1}, \quad \beta=\beta_{0} a^{s} \phi^{m(s)}, \quad F(\phi)=D(s) \phi^{2}, \quad V(\phi)=\lambda \phi^{2 p(s)},
$$

where

$$
D(s)=\frac{(2 s+3)^{2}}{48(s+1)(s+2)}, \quad p(s)=\frac{3(s+1)}{2 s+3}, \quad m(s)=\frac{2 s^{2}+6 s+3}{2 s+3},
$$

and $s, \lambda$ are free parameters. The change of variables (8) gives

$$
w=\sigma_{0} a^{3} \phi^{2 p(s)}, \quad z=\frac{3}{\beta_{0} \chi(s)} a^{-s} \phi^{1-m(s)},
$$

where $\sigma_{0}$ is an integration constant and $\chi(s)=-6 s /(2 s+3)$, Lagrangian (22) becomes, for $k=0$,

$$
\mathcal{L}=\gamma(s) w^{s / 3} \dot{z} \dot{w}-\lambda w
$$

where $z$ is cyclic and $\gamma(s)=(2 s+3) /\left[12 \sigma_{0}^{2}(s+2)(s+1)\right]$. The conjugate momenta are

$$
\pi_{z}=\frac{\partial \mathcal{L}}{\partial \dot{z}}=\gamma(s) w^{s / 3} \dot{w}, \quad \pi_{w}=\frac{\partial \mathcal{L}}{\partial \dot{w}}=\gamma(s) w^{s / 3} \dot{z},
$$

and the Hamiltonian is

$$
\tilde{\mathcal{H}}=\frac{\pi_{z} \pi_{w}}{\gamma(s) w^{s / 3}}+\lambda w .
$$

The Noether symmetry is given by $\pi_{z}=\Sigma_{0}$. Quantizing Eqs. (32), we get

$$
\pi \longrightarrow-i \partial_{z}, \quad \pi_{w} \longrightarrow-i \partial_{w}
$$

and then the WDW equation

$$
\left[\left(i \partial_{z}\right)\left(i \partial_{w}\right)+\tilde{\lambda} w^{1+s / 3}\right] \mid \Psi>=0,
$$

where $\tilde{\lambda}=\gamma(s) \lambda$. The quantum version of Eqs. (32) give

$$
-i \partial_{z}\left|\Psi>=\Sigma_{0}\right| \Psi>
$$

so that dynamics results reduced. A straightforward integration of Eqs. (35) and (36) gives

$$
|\Psi>=| \Omega(w)>\mid \chi(z)>\propto e^{i \Sigma_{0} z} e^{-i \tilde{\lambda} w^{2+s / 3}},
$$

which is an oscillating wave function and the Hartle criterion is recovered. In the semi-classical limit, we have two first integrals of motion: $\Sigma_{0}$ (i.e. the equation for $\pi_{z}$ ) and $E_{\mathcal{L}}=0$,i.e. the Hamiltonian (33) which becomes the equation for $\pi_{w}$. Classical trajectories in the configuration space $\tilde{Q} \equiv\{w, z\}$ are immediately recovered

$$
w(t)=\left[k_{1} t+k_{2}\right]^{3 /(s+3)}, \quad z(t)=\left[k_{1} t+k_{2}\right]^{(s+6) /(s+3)}+z_{0},
$$

then, going back to $Q \equiv\{a, \phi\}$, we get the classical cosmological behaviour

$$
a(t)=a_{0}\left(t-t_{0}\right)^{l(s)}, \quad \phi(t)=\phi_{0}\left(t-t_{0}\right)^{q(s)}, \quad l(s)=\frac{2 s^{2}+9 s+6}{s(s+3)}, \quad q(s)=-\frac{2 s+3}{s},
$$

which means that Hartle criterion selects classical universes. Depending on the value of $s$, we get Friedman, power-law, or pole-like behaviors. Finally, if we take into account generic Bianchi models, the configuration space is $Q \equiv\left\{a_{1}, a_{2}, a_{3}, \phi\right\}$ and more than one symmetry can exist as it is shown in [10]. The considerations on the oscillatory regime of the wave function of the universe and the recovering of classical behaviors are exactly the same. 


\subsection{Fourth-Order Gravity Cosmologies}

Similar arguments work for higher-order gravity minisuperspaces. In particular, let us consider fourth-order gravity given by the action

$$
\mathcal{S}=\int d^{4} x \sqrt{-g} f(R)
$$

where $f(R)$ is a generic function of scalar curvature. If $f(R)=R+2 \Lambda$, the standard second-order Einstein gravity is recovered. We are discarding matter contributions for the sake of simplicity. Reducing the action to a point-like, FRW one, we have to write

$$
\mathcal{S}=\int d t \mathcal{L}(a, \dot{a} ; R, \dot{R})
$$

where dot means derivative with respect to the cosmic time. The scale factor $a$ and the Ricci scalar $R$ are the canonical variables. This position could seem arbitrary since $R$ depends on $a, \dot{a}, \ddot{a}$, but it is generally used in canonical quantization $[15,16]$. The definition of $R$ in terms of $a, \dot{a}, \ddot{a}$ introduces a constraint which eliminates second and higher order derivatives in action (41), and yields to a system of second order differential equations in $\{a, R\}$. Action (41) can be written as

$$
\mathcal{S}=2 \pi^{2} \int d t\left\{a^{3} f(R)-\lambda\left[R+6\left(\frac{\ddot{a}}{a}+\frac{\dot{a}^{2}}{a^{2}}+\frac{k}{a^{2}}\right)\right]\right\},
$$

where the Lagrange multiplier $\lambda$ is derived by varying with respect to $R$. It is $\lambda=a^{3} f^{\prime}(R)$. Here prime means derivative with respect to $R$. To recover the analogy with previous scalar-tensor models, let us introduce the auxiliary field $p \equiv f^{\prime}(R)$, so that the Lagrangian in (42) becomes

$$
\mathcal{L}=6 a \dot{a}^{2} p+6 a^{2} \dot{a} \dot{p}-6 k a p-a^{3} W(p),
$$

which is of the same form of (22) a part the kinetic term; $a, p$ are independent fields. The potential $W(p)$ is defined as

$$
W(p)=h(p) p-r(p)
$$

where

$$
r(p)=\int f^{\prime}(R) d R=\int p d R=f(R), \quad h(p)=R,
$$

such that $h=\left(f^{\prime}\right)^{-1}$ is the inverse function of $f^{\prime}$. The configuration space is now $Q \equiv\{a, p\}$ and $p$ has the same role of the above $\phi$. Condition (5) is now realized by the vector field

$$
X=\alpha(a, p) \frac{\partial}{\partial a}+\beta(a, p) \frac{\partial}{\partial p}+\dot{\alpha} \frac{\partial}{\partial \dot{a}}+\dot{\beta} \frac{\partial}{\partial \dot{p}},
$$

and explicitly gives the system

$$
\begin{aligned}
& p\left[\alpha+2 a \frac{\partial \alpha}{\partial a}\right] p+a\left[\beta+a \frac{\partial \beta}{\partial a}\right]=0, \\
& a^{2} \frac{\partial \alpha}{\partial p}=0, \\
& 2 \alpha+a \frac{\partial \alpha}{\partial a}+2 p \frac{\partial \alpha}{\partial p}+a \frac{\partial \beta}{\partial p}=0, \\
& 6 k[\alpha p+\beta a]+a^{2}\left[3 \alpha W+a \beta \frac{\partial W}{\partial p}\right]=0 .
\end{aligned}
$$


The solution of this system, i.e. the existence of a Noether symmetry, gives $\alpha, \beta$ and $W(p)$. It is satisfied for $\alpha=\alpha(a)$, and $\beta(a, p)=\beta_{0} a^{s} p$ where $s$ is a parameter and $\beta_{0}$ is an integration constant. In particular,

$$
\begin{gathered}
s=0 \longrightarrow \alpha(a)=-\frac{\beta_{0}}{3} a, \quad \beta(p)=\beta_{0} p, \quad W(p)=W_{0} p, \quad k=0, \\
s=-2 \longrightarrow \alpha(a)=-\frac{\beta_{0}}{a}, \quad \beta(a, p)=\beta_{0} \frac{p}{a^{2}}, \quad W(p)=W_{1} p^{3}, \quad \forall k,
\end{gathered}
$$

where $W_{0}$ and $W_{1}$ are constants. Let us discuss separately the solutions (51) and (52).

In the case $s=0$, the induced change of variables $Q \equiv\{a, p\} \longrightarrow \tilde{Q} \equiv\{w, z\}$ can be $w(a, p)=a^{3} p$, and $z(p)=\ln p$. Lagrangian (43) becomes

$$
\tilde{\mathcal{L}}(w, \dot{w}, \dot{z})=\dot{z} \dot{w}-2 w \dot{z}^{2}+\frac{\dot{w}^{2}}{w}-3 W_{0} w
$$

and, obviously, $z$ is the cyclic variable. The conjugate momenta are

$$
\pi_{z} \equiv \frac{\partial \tilde{\mathcal{L}}}{\partial \dot{z}}=\dot{w}-4 \dot{z}=\Sigma_{0}, \quad \pi_{w} \equiv \frac{\partial \tilde{\mathcal{L}}}{\partial \dot{w}}=\dot{z}+2 \frac{\dot{w}}{w} .
$$

and the Hamiltonian is

$$
\mathcal{H}\left(w, \pi_{w}, \pi_{z}\right)=\pi_{w} \pi_{z}-\frac{\pi_{z}^{2}}{w}+2 w \pi_{w}^{2}+6 W_{0} w
$$

By canonical quantization, reduced dynamics is given by

$$
\left[\partial_{z}^{2}-2 w^{2} \partial_{w}^{2}-w \partial_{w} \partial_{z}+6 W_{0} w^{2}\right]\left|\Psi>=0, \quad-i \partial_{z}\right| \Psi>=\Sigma_{0} \mid \Psi>
$$

However, we have assumed a simple factor ordering choice in the WDW equation (56). Immediately, the wave function has an oscillatory factor, being $\left|\Psi>\sim e^{i \Sigma_{0} z}\right| \chi(w)>$. The function $\mid \chi>$ satisfies the Bessel differential equation

$$
\left[w^{2} \partial_{w}^{2}+i \frac{\Sigma_{0}}{2} w \partial_{w}+\left(\frac{\Sigma_{0}^{2}}{2}-3 W_{0} w^{2}\right)\right] \chi(w)=0,
$$

whose solutions are linear combinations of Bessel functions $Z_{v}(w)$

$$
\chi(w)=w^{1 / 2-i \Sigma_{0} / 4} Z_{v}(\Lambda w), \quad \text { where } \quad v= \pm \frac{1}{4} \sqrt{4-9 \Sigma_{0}^{2}-i 4 \Sigma_{0}}, \quad \Lambda= \pm 9 \sqrt{\frac{W_{0}}{2}} .
$$

The oscillatory regime for this component depends on the reality of $v$ and $\Lambda$. The wave function of the universe, from Noether symmetry (51) is then

$$
\Psi(z, w) \sim e^{i \Sigma_{0}[z-(1 / 4) \ln w]} w^{1 / 2} Z_{v}(\Lambda w) .
$$

For large $w$, the Bessel functions have an exponential behavior, so that the wave function (59) can be written as $\Psi \sim e^{i\left[\Sigma_{0} z-\left(\Sigma_{0} / 4\right) \ln w \pm \Lambda \omega\right]}$. Due to the oscillatory behaviour of $\Psi$, Hartle's criterion is immediately recovered. By identifying the exponential factor with $S_{0}$, we can recover the conserved momenta $\pi_{z}, \pi_{w}$ and select classical trajectories. Going back to the old variables, we get the cosmological solutions

$$
a(t)=a_{0} e^{(\Lambda / 6) t} \exp \left\{-\frac{z_{1}}{3} e^{-(2 \Lambda / 3) t}\right\}, \quad p(t)=p_{0} e^{(\Lambda / 6) t} \exp \left\{z_{1} e^{-(2 \Lambda / 3) t}\right\}
$$


where $a_{0}, p_{0}$ and $z_{1}$ are integration constants. It is clear that $\Lambda$ plays the role of a cosmological constant and inflationary behavior is asymptotically recovered.

A similar analysis can be pursued for the case $s=-2$. Here, the new variables adapted to the foliation for the solution (52) are $w(a, p)=a p$ and $z(a)=a^{2}$. Going over the same steps as above, the wave function of the universe is given by

$$
\Psi(z, w) \sim e^{i\left[\Sigma_{1} z+9 k w^{2}+\left(3 W_{1} / 4\right) w^{4}\right]},
$$

and the classical cosmological solutions are

$$
a(t)= \pm \sqrt{h(t)}, \quad p(t)= \pm \frac{c_{1}+\left(\Sigma_{1} / 3\right) t}{\sqrt{h(t)}}
$$

where

$$
h(t)=\left(\frac{W_{1} \Sigma_{1}^{3}}{36}\right) t^{4}+\left(\frac{W_{1} w_{1} \Sigma_{1}}{6}\right) t^{3}+\left(k \Sigma_{1}+\frac{W_{1} w_{1}^{2} \Sigma_{1}}{2}\right) t^{2}+w_{1}\left(6 k+W_{1} w_{1}^{2}\right) t+z_{2} .
$$

$w_{1}, z_{1}$ and $z_{2}$ are integration constants. Immediately we see that, for large $t, a(t) \sim t^{2}$, and $p(t) \sim t^{-1}$, which is a power-law inflationary behavior. An extensive discussion of Noether symmetries in $f(R)$ gravity is given in [13].

\section{Discussion and Conclusions}

A main role in QC is played by the identification of conserved quantities that give rise to peaked behaviors in the wave function of the universe. Such a function is the solution of the WDW equation, the corresponding of Schrödinger equation in Quantum Mechanics. Peaked behaviors means correlations among variables and then the possibility to obtain classical universes according to the Hartle interpretative criterion. These conserved quantities can naturally be related to the Noether symmetries of the theory. The existence of symmetries depends, in several cases, by the identification of suitable Lagrange multipliers that allow to recast the point-like Lagrangian of the given minisuperspace model in a canonical form [12]. In this sense, the Noether symmetries can be considered as "constraints" of the theory that allow to reduce the dynamics and recover classical solutions. Reversing the argument, if the wave function of the universe is related to the probability to get a classical cosmological solution, the existence of Noether symmetries tell us when the Hartle criterion works. Some remarks are necessary at this point. First of all, we have to stress that the wave function is only related to the probability to get a certain behavior but it is not the probability amplitude since, till now, QC is not a unitary theory. Furthermore, the Hartle criterion works in the context of an Everett-type interpretation of QC [17] which assumes the ideas that the universe branches into a large number of copies of itself whenever a measurement is made. This point of view is called Many Worlds interpretation of Quantum Mechanics. Such an interpretation is just one way of thinking and gives a formulation of Quantum Mechanics designed to deal with correlations internal to individual, isolated systems. The Hartle criterion gives an operative interpretation of such correlations. In particular, if the wave function is strongly peaked in some region of configuration space, we predict that we will observe the correlations which characterize that region. On the other hand, if the wave function is smooth in some region, we predict that correlations which characterize that region are precluded to the observations. If the wave function is neither peaked nor smooth, no predictions are possible from observations. In other words, we can read the correlation of some region of minisuperspace as causal connection. 
What we proposed here is a criterion by which the Hartle point of view can be recovered without arbitrariness. If a Noether symmetry (or more than one) is present for a given minisuperspace model, then strongly peaked (oscillatory) subsets of the wave function of the universe are found. Vice-versa, oscillatory parts of the wave function can be always connected to conserved momenta and then to Noether symmetries. From a general point of view, this is the same philosophy of many branches of physics: finding symmetries allows to solve dynamics, gives the main features of systems and simplifies the interpretation of results. We have worked out this approach for minisuperspace models coming from Extended Theories of Gravity showing that identification of suitable Noether symmetries allows to completely solve the dynamical system.

\section{References}

[1] C.W. Misner, Phys. Rev. 186, 1319 (1969).

[2] B.S. DeWitt, Phys. Rev. 160, 1113 (1967).

[3] J.B. Hartle, in Gravitation in Astrophysics, eds. S. Carter et al. Plenum, NY (1986).

[4] J.J. Halliwell, Nucl. Phys. B 266, 228 (1986).

[5] S. Capozziello, M. De Laurentis, Phys. Repts., 509, 167 (2011).

[6] N.D. Birrell, P.C.W. Davies, Quantum Fields in Curved Space (Cambridge Univ. Press, 1982).

[7] A.A. Starobinsky, Phys. Lett. B 91 (1980) 99.

[8] S. Capozziello, Int. J. Mod. Phys. D 11, 483 (2002).

[9] S. Capozziello, R. de Ritis, C. Rubano and P. Scudellaro, La Riv. del Nuovo Cim. 4, 1 (1996).

[10] S. Capozziello, G. Marmo, C. Rubano, and P. Scudellaro, Int. J. Mod. Phys. D4, 491 (1997).

[11] S. Capozziello, G. Lambiase, Gen. Rel. Grav. 32673 (2000).

[12] S. Capozziello, M. De Laurentis, S.D. Odintsov, Eur.Phys. J. C 72, 2068 (2012).

[13] S. Capozziello, A. De Felice, JCAP 0808, 016, (2008).

[14] S. Capozziello and R. de Ritis, Phys. Lett. A 177, 1 (1993).

[15] A. Vilenkin, Phys. Rev. D 32, 2511 (1985).

[16] S. Capozziello, R. de Ritis, and A.A. Marino, Gen. Rel. Grav. 30, 1247 (1998).

[17] H. Everett, Rev. Mod. Phys. 29, 454 (1957). 